\title{
Editorial
}

\section{Prime Minister's prize for improving the repair of human tissue, a beacon for wound research in Australia}

\section{Cowin AJ}

For referencing Cowin AJ. 2021 Prime Minister's prize for improving the repair of human tissue, a beacon for wound research in Australia. Wound Practice and Research 2021; 29(4):185.

DOI https://doi.org/10.33235/wpr.29.4.185

The broad purpose of laboratory-based wound research is to obtain a scientific understanding of the mechanistic processes that underpin normal and abnormal wound healing. Translation of this knowledge can lead to the development of new tools, technologies, therapeutics diagnostics and sensors that ultimately will lead to improved wound management and patient care. While this process may seem far removed from the day-to-day challenge of dealing with patients with impaired healing, occasionally we see breakthroughs and achievements that encourage both researchers and clinicians alike.

One such achievement was highlighted this month when Professor Tony Weiss AM from the University of Sydney, was awarded the 2021 Prime Minister's Prize for Innovation. For the past two decades, Professor Weiss has pioneered global research into tropoelastin and elastic fibres which give human skin its elasticity. He initiated the development of tropoelastin for a range of medical applications, due to its unique physical elastic properties and ability to support cell growth and tissue repair. Clinical trials have demonstrated the biocompatability and safety of this synthetic human elastin

Prof Allison J Cowin

Editor Wound Practice and Research and elastin-based synthetic skin scaffolds have been tested in repairing full thickness skin wounds. In 2008, Professor Weiss founded the company Elastagen to commercialise his research and inventions. The company raised $\$ 35$ million in venture capital and grant funding, completed clinical trials and scaled-up production. Ten years later, Elastagen was sold to AbbVie, one of the world's largest biopharmaceutical companies for \$334 million - one of the largest transactions ever completed in Australia's life science sector.

Clearly, we have the talent and capability to perform world-class wound research in Australia but innovation and translation is often limited by a lack of focus and funding. In order to understand the breadth of research being undertaken in Australia and identify gaps in knowledge and areas of strengths, the Medical Research Future Fund Rapid Research Translation Program has supported a series of scoping reviews to identify the type and level of wound research that has been performed over the past 10 years. Two of the review protocols by Bui and colleagues are published in this issue of the journal and we will look forward to reading the outcomes of these scoping reviews in due course. With the right support, there is hope that future Prime Minister's prizes will be awarded for wound research but more importantly that this research leads to improved ways to treat impaired healing for all Australians.

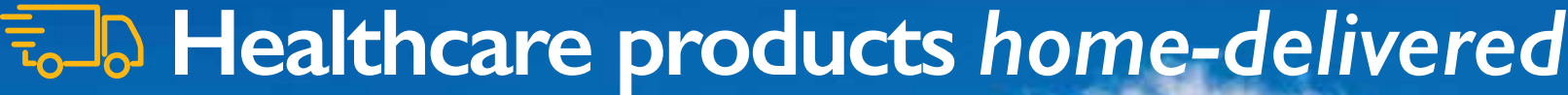

We offer a comprehensive range in:

- Continence

- Respiratory

- Wound care

- Mobility aids and equipment

- Skin care

- Pressure management and bedding

- Nutrition

Call us or visit our website:

(c) 1300886601

@ orders@brightsky.com.au

(U) www.brightsky.com.au
All proceeds from BrightSky go towards helping people with physical disability and their support network

www.brightsky.com.au

\section{Supporting NDIS \\ participants since 2013}

